\title{
Risk Factors of Symptomatic Intracerebral Hemorrhage After tPA Therapy for Acute Stroke
}

\author{
Maarten G. Lansberg, MD, PhD, Vincent N. Thijs, MD, PhD, Roland Bammer, PhD, \\ Stephanie Kemp, BS, Christine A.C. Wijman, MD, Michael P. Marks, MD, Gregory W. \\ Albers, MD, and on behalf of the DEFUSE Investigators \\ Stanford Stroke Center (M.G.L., R.B., S.K., C.A.C.W., M.P.M., G.W.A.), Stanford University \\ Medical Center, Palo Alto, Calif; and the Department of Neurology (V.N.T.), University Hospitals \\ of Leuven, Belgium
}

\begin{abstract}
Background-Studies evaluating predictors of tPA-associated symptomatic intracerebral hemorrhage (SICH) have typically focused on clinical and CT-based variables. MRI-based variables have generally not been included in predictive models, and little is known about the influence of reperfusion on SICH risk.
\end{abstract}

\begin{abstract}
Methods-Seventy-four patients were prospectively enrolled in an open-label study of intravenous tPA administered between 3 and 6 hours after symptom onset. An MRI was obtained before and 3 to 6 hours after tPA administration. The association between several clinical and MRI-based variables and tPA-associated SICH was determined using multivariate logistic regression analysis. SICH was defined as a $\geq 2$ point change in National Institutes of Health Stroke Scale Score (NIHSSS) associated with any degree of hemorrhage on CT or MRI. Reperfusion was defined as a decrease in PWI lesion volume of at least $30 \%$ between baseline and the early followup MRI.
\end{abstract}

\begin{abstract}
Results-SICH occurred in 7 of 74 (9.5\%) patients. In univariate analysis, NIHSSS, DWI lesion volume, PWI lesion volume, and reperfusion status were associated with an increased risk of SICH $(P<0.05)$. In multivariate analysis, DWI lesion volume was the single independent baseline predictor of SICH (odds ratio 1.42; 95\% CI 1.13 to 1.78 per $10 \mathrm{~mL}$ increase in DWI lesion volume). When early reperfusion status was included in the predictive model, the interaction between DWI lesion volume and reperfusion status was the only independent predictor of SICH (odds ratio 1.77 ; $95 \%$ CI 1.25 to 2.50 per $10 \mathrm{~mL}$ increase in DWI lesion volume).
\end{abstract}

Conclusion-Patients with large baseline DWI lesion volumes who achieve early reperfusion appear to be at greatest risk of SICH after tPA therapy.

(C) 2007 American Heart Association, Inc.

Correspondence Maarten G. Lansberg, MD, PhD, Stanford University, Stanford Stroke Center, 701 Welch Road, Suite B 325, Palo Alto, California 94304. Lansberg@ stanford.edu.

Disclosures M.G.L., R.B., and G.W.A. received NIH grant funding related to this research. 


\section{Keywords}

ischemic stroke; acute treatment; plasminogen activator; perfusion-weighted MRI; diffusionweighted MRI; reperfusion; intracerebral hemorrhage; outcome

The most feared complication of tissue plasminogen activator (tPA) therapy for acute stroke patients is symptomatic intracerebral hemorrhage (SICH). Gaining insight into the riskfactors that are associated with intracerebral hemorrhage may lead to interventions that reduce the risk of SICH and improve patient selection for acute stroke trials. Identification of SICH risk factors could be of particular importance in patients treated with tPA beyond the established 3-hour time-window as the overall benefit of tPA diminishes with increasing duration of the "symptom onset-to-treatment" time. ${ }^{1}$ Several previous studies have investigated the relationship between clinical, radiological, and laboratory variables and SICH in tPA-treated stroke-patients. The potential role of MRI in predicting SICH after tPA has not yet been carefully examined because most previous studies did not have baseline MRI data on tPA-treated stroke patients. Similarly, the influence of early reperfusion on the development of SICH is poorly understood because early reperfusion data in acute stroke patients are relatively sparse. We were able to study these associations using data from the Diffusion and Perfusion Imaging Evaluation For Understanding Stroke Evolution (DEFUSE) study in which multimodal MRI was obtained both before and soon after tPA therapy. Specifically, this substudy of DEFUSE aims to identify independent baseline predictors (both clinical and MRI-based) of SICH and evaluate whether reperfusion plays a role in the development of SICH.

\section{Methods}

Data were included from all patients enrolled in the Diffusion and Perfusion Imaging Evaluation For Understanding Stroke Evolution (DEFUSE) study. ${ }^{2}$ This is a multi-center open-label study of intravenous tPA administered during the 3- to 6-hour time-window after stroke onset. Details of the study design and results of the primary hypotheses have been reported previously. ${ }^{2}$ Briefly, patients were eligible if they had a clinical syndrome consistent with stroke, a National Institutes of Health Stroke Scale Score (NIHSSS) $>5$, and no evidence of acute hemorrhage or clearly identifiable hypodensity involving more than $1 / 3$ of the MCA distribution on pretreatment CT. Enrolled patients underwent an MRI, including diffusion-weighted imaging (DWI) and perfusion-weighted imaging (PWI), before and 3 to 6 hours after tPA administration $(0.9 \mathrm{mg} / \mathrm{kg})$. Patients who developed worsening of 2 or more points on the NIHSS underwent an urgent CT or MRI. If any degree of brain hemorrhage was detected, an independent Data Safety and Monitoring Board (DSMB) reviewed the clinical and imaging data. The DSMB classified symptomatic hemorrhages as "minor" if they were associated with 2 to 3 points of worsening on the NIHSS within 36 hours of tPA administration and "major" if they were associated with 4 or more points of worsening within 36 hours of tPA administration. For all patients who had a SICH, the baseline CT scan was read by a blinded neuroradiologist (M.P.M.) to determine whether there was clearly identifiable hypodensity involving more than $1 / 3$ of the middle cerebral 
artery (MCA) territory. The study protocol was approved by the local institutional review board at each participating center.

Baseline variables that were obtained for each patient included: age, gender, history of cardiac disease, smoking, diabetes, stroke/ TIA, hypertension and hyperlipidemia, time to treatment, serum glucose, platelet count, systolic blood pressure at admission, diastolic blood pressure at admission, NIHSSS, DWI lesion volume, and PWI lesion volume. Reperfusion was defined according to predetermined DEFUSE study criteria as a reduction of PWI lesion volume between the baseline and follow-up MRI of at least $10 \mathrm{~mL}$ and at least $30 \%$. Only patients who had technically adequate baseline and follow-up PWI scans were included in the reperfusion analysis. Patients who had a PWI lesion volume $<10 \mathrm{~mL}$ at baseline were excluded from the reperfusion analysis as these patients, by definition, could not achieve reperfusion. The method for assessing MRI lesion volumes has been described previously. $^{2}$

Differences in baseline characteristics between patients with and without SICH were assessed using the $t$ test for continuous variables with a normal distribution, the Wilcoxon rank-sum test for variables that are not normally distributed, and the chi-square test for categorical variables. The association between reperfusion (present or absent) and SICH was determined with the chi-square test. Variables that reached a $P<0.1$ in univariate analysis were included in a multivariate forward stepwise regression analysis. Two separate models were generated. The first model included only baseline variables. A second model included both baseline variables as well as interaction terms between each of the baseline variables and reperfusion status. Separate models were constructed with any SICH and major SICH as the dependent variable. Variables were deemed statistically significant in the multivariate model at a $P<0.05$. All statistical analyses were performed in SPSS version 13.0.

\section{Results}

Seven of the 74 patients (9.5\%) experienced a SICH (4 major and 3 minor). Three of the 4 major SICH patients (75\%) died. Two died during the acute hospitalization and 1 at 109 days. Of the 3 patients with minor SICH, 1 died (33\%).

The Table displays the baseline variables for patients who developed SICH and for patients who did not. NIHSSS, PWI lesion volume, and DWI lesion volume are significantly different between these 2 groups $(P<0.05)$. In addition, rate of reperfusion between the baseline and 3- to 6-hour follow-up MRI scan (early reperfusion) was greater in patients with SICH than in those without $(P=0.04)$. When only baseline variables were entered into the multivariate logistic regression model, DWI lesion volume was the only independent predictor of SICH (odds ratio 1.42; 95\% CI 1.13 to 1.78 per $10 \mathrm{~mL}$ change in DWI lesion volume). This was also the case when the analysis was limited to patients with major SICH (odds ratio 1.52 ; $95 \%$ CI 1.14 to 2.03 per $10 \mathrm{~mL}$ change in DWI lesion volume). When baseline variables, reperfusion status, and the interaction terms between baseline variables and reperfusion status were included, the interaction between baseline DWI lesion volume and reperfusion status remained as the single independent risk factor of any SICH (odds ratio $1.77 ; 95 \%$ CI 1.25 to 2.50 per $10 \mathrm{~mL}$ change in DWI lesion volume) and major SICH 
(odds ratio $1.90 ; 95 \%$ CI 1.21 to 3.00 per $10 \mathrm{~mL}$ change in DWI lesion volume). Patients with major SICH typically had baseline DWI volumes $>90 \mathrm{~mL}$. The only exception was a patient who developed SICH during a carotid endarterectomy performed the day after the stroke (see Figure). Both major and minor hemorrhages occurred almost exclusively in patients who experience early reperfusion. (see Figure) The baseline DWI volumes in the 4 patients with major SICH were 105, 103, 94, and $22 \mathrm{~mL}$; the volumes in the 3 patients with minor SICH were 65,34 , and $12 \mathrm{~mL}$. Only 1 of the baseline CT scans of patients with SICH showed evidence of clearly identifiable hypodensity involving more than $1 / 3$ of the MCA territory. This patient had a major hemorrhage 4 hours after tPA treatment and died on day 1.

\section{Discussion}

In the DEFUSE study population, DWI lesion volume was the single independent baseline predictor of SICH among multiple clinical and laboratory baseline variables assessed. In addition, there was a significant interaction between DWI volume and early reperfusion, indicating a synergistic effect between DWI lesion volume and early reperfusion on the risk of SICH; patients who had large DWI lesion volumes and achieved early reperfusion were at particularly high risk of SICH.

The following variables have been identified as independent risk factors for SICH after thrombolysis in previous studies: Early infarct signs on pretreatment $\mathrm{CT},{ }^{3-8}$ elevated serum glucose or history of diabetes, ${ }^{4-6,9-11}$ symptom severity, ${ }^{7,12}$ advanced age, ${ }^{8}$ increased time to treatment, ${ }^{11}$ high systolic blood pressure, ${ }^{13}$ low platelet count, ${ }^{4}$ history of congestive heart failure, ${ }^{8}$ and low plasminogen activator inhibitor (PAI-1) levels. ${ }^{14}$ Our finding that DWI volume is an independent predictor of SICH is in accord with previous reports that have shown that the extent of early infarct signs on CT is a predictor of SICH because both large DWI lesions and widespread CT hypodensity are markers of the volume of severe cerebral ischemia. As none of the previous studies obtained MRI data before tPA administration, it is unknown whether the variables that were identified as independent predictors of SICH in those studies would have remained as independent predictors if DWI and PWI lesion volume had been included in the predictive models. Because DEFUSE excluded patients with hypodensity involving more than $1 / 3$ of the MCA territory on CT, we are unable to assess the influence of major early infarct signs on CT in this study. Of note, only one of the DEFUSE SICH patients was found in retrospect to have major early infarct signs on the baseline CT (a protocol violation).

Our results are in accord with a study by Selim et al, who demonstrated that the volume of the baseline DWI lesion with ADC $550 \times 10^{-6} \mathrm{~mm}^{2} / \mathrm{s}$ is an independent predictor of any postthrombolysis hemorrhage. ${ }^{15}$ There are several differences between our study and Selim's; we restricted our analysis to patients with SICH, we included baseline PWI data and reperfusion data in the predictive model, all patients in the DEFUSE study were treated in the 3- to 6-hour time-window, and we did not quantify the ADC values of the DWI lesion in this study. Selim's finding that the depth of ischemia measured by ADC values may be important in predicting hemorrhagic transformation after thrombolysis is in agreement with another study that showed an association between low ADC and hemorrhagic transformation 
in stroke patients not treated with thrombolysis. ${ }^{16}$ The aim of this study was to evaluate the predictive value of baseline clinical and easily obtainable baseline MRI variables (DWI and PWI lesion volumes). A separate study is underway to determine whether quantitative ADC data and PWI severity parameters can be used to refine the prediction of SICH as well as any hemorrhage (hemorrhagic transformation and SICH) after tPA.

Little is known about the relationship between reperfusion and the development of SICH in patients treated with intravenous tPA. It has been postulated that reperfusion-related damage to the ischemic microvasculature may contribute to SICH risk. ${ }^{17,18}$ Our findings support this view as reperfusion, particularly in patients with large baseline DWI lesion volumes, was associated with an increased SICH risk. Studies of intraarterial thrombolytics have not demonstrated an increased risk of SICH in patients who recanalize; however, these studies did not include an assessment of baseline DWI volume. ${ }^{10,19,20}$ Although our data require validation in future studies, it appears that exclusion of patients with large DWI lesion volumes could improve the safety and efficacy of reperfusion therapies in the 3- to 6-hour time-window.

\section{Acknowledgments}

Sources of Funding The funding for this study was provided by NIH grants K23 NS051372, Principal Investigator Maarten G. Lansberg; RO1 NS39325, Principal Investigator, Gregory W. Albers; and K24 NS044848, Principal Investigator, Gregory W. Albers.

\section{Appendix}

The DEFUSE Investigators are: Stanford University: Gregory Albers, MD, Roland Bammer, $\mathrm{PhD}$, Anna Caulfield Finley, MD, Scott Hamilton, PhD, Wataru Kakuda, MD, Stephanie Kemp, BS, Maarten G. Lansberg, MD, PhD, Michael Marks, MD, Michael Moseley, PhD, Neil E. Schwartz, MD, PhD, Christine A.C. Wijman, MD; University of Pittsburgh: Lawrence Wechsler, MD, Sharon De-Cesare; University of Gasthuisberg: Vincent Thijs, MD, Ingeborg Wauters, RN; Beth Israel Deaconess: Gottfried Schlaug, MD, Cheryl Hogan; University of Alberta: Ashfaq Shuaib, MD, Karen Kastelic, RN; University of Utah: Elaine Skalabrin, MD, Janet Woodruff, RN; and Wayne State University: William Coplin, MD, Flicia Mada, RN. Data Safety Monitoring Board: Jeffrey Saver, MD (Chair), Pierre Fayed, MD, Janet Wilterdink, MD, Scott Hamilton, PhD.

\section{References}

1. Hacke W, Donnan G, Fieschi C, Kaste M, von Kummer R, Broderick J, Brott T, Frankel M, Grotta J, Haley EC, Kwiatkowski T, Levine SR, Lewandowski C, Lu M, Lyden P, Marler JR, Patel S, Tilley BC, Albers GW, Bluhmki E, Wilhelm M, Hamilton S, ATLANTIS Trials Investigators; ECASS Trials Investigators; NINDS rt-PA Study Group Investigators. Association of outcome with early stroke treatment: pooled analysis of ATLANTIS, ECASS, and NINDS rt-PA stroke trials. Lancet. 2004; 363:768-774. [PubMed: 15016487]

2. Albers GW, Thijs VN, Wechsler L, Kemp S, Schlaug G, Skalabrin E, Bammer R, Kakuda W, Lansberg MG, Shuaib A, Coplin W, Hamilton S, Moseley M, Marks MP, for the DEFUSE Investigators. Magnetic resonance imaging profiles predict clinical response to early reperfusion: the diffusion and perfusion imaging evaluation for understanding stroke evolution (defuse) study. Ann Neurol. 2006; 60:508-517. [PubMed: 17066483] 
3. Intracerebral hemorrhage after intravenous t-PA therapy for ischemic stroke. The NINDS and Stroke rt-PA Stroke Study Group. Stroke. 1997; 28:2109-2118. [PubMed: 9368550]

4. Tanne D, Kasner SE, Demchuk AM, Koren-Morag N, Hanson S, Grond M, Levine SR. Markers of increased risk of intracerebral hemorrhage after intravenous recombinant tissue plasminogen activator therapy for acute ischemic stroke in clinical practice: the multicenter rt-pa stroke survey. Circulation. 2002; 105:1679-1685. [PubMed: 11940547]

5. Jaillard A, Cornu C, Durieux A, Moulin T, Boutitie F, Lees KR, Hommel M. Hemorrhagic transformation in acute ischemic stroke. The MAST-E study. MAST-E group. Stroke. 1999; 30:1326-1332. [PubMed: 10390303]

6. Barber PA, Demchuk AM, Zhang J, Buchan AM. Validity and reliability of a quantitative computed tomography score in predicting outcome of hyperacute stroke before thrombolytic therapy. ASPECTS Study Group. Alberta Stroke Programme Early CT Score. Lancet. 2000; 355:1670-1674. [PubMed: 10905241]

7. Dubey N, Bakshi R, Wasay M, Dmochowski J. Early computed tomography hypodensity predicts hemorrhage after intravenous tissue plasminogen activator in acute ischemic stroke. $\mathrm{J}$ Neuroimaging. 2001; 11:184-188. [PubMed: 11296590]

8. Larrue V, von Kummer R, Muller A, Bluhmki E. Risk factors for severe hemorrhagic transformation in ischemic stroke patients treated with recombinant tissue plasminogen activator : a secondary analysis of the European-Australasian Acute Stroke Study (ECASS II). Stroke. 2001; 32:438-441. [PubMed: 11157179]

9. Demchuk AM, Morgenstern LB, Krieger DW, Chi T Linda, Hu W, Wein TH, Hardy RJ, Grotta JC, Buchan AM. Serum glucose level and diabetes predict tissue plasminogen activator-related intracerebral hemorrhage in acute ischemic stroke. Stroke. 1999; 30:34-39. [PubMed: 9880385]

10. Kase CS, Furlan AJ, Wechsler LR, Higashida RT, Rowley HA, Hart RG, Molinari GF, Frederick LS, Roberts HC, Gebel JM, Sila CA, Schulz GA, Roberts RS, Gent M. Cerebral hemorrhage after intra-arterial thrombolysis for ischemic stroke: The PROACT II Trial. Neurology. 2001; 57:16031610. [PubMed: 11706099]

11. Hill MD, Buchan AM, for The Canadian Alteplase for Stroke Effectiveness Study (CASES) Investigators. Thrombolysis for acute ischemic stroke: Results of the Canadian Alteplase for Stroke Effectiveness Study. CMAJ. 2005; 172:1307-1312. [PubMed: 15883405]

12. Symptomatic intracerebral hemorrhage after t-pa for stroke. The National Institute of Neurological Disorders and Stroke rt-PA Stroke Study Group. Stroke. 1997; 28:210.

13. Gilligan AK, Markus R, Read S, Srikanth V, Hirano T, Fitt G, Arends M, Chambers BR, Davis SM, Donnan GA. Baseline blood pressure but not early computed tomography changes predicts major hemorrhage after streptokinase in acute ischemic stroke. Stroke. 2002; 33:2236-2242. [PubMed: 12215593]

14. Ribo M, Montaner J, Molina CA, Arenillas JF, Santamarina E, Quintana M, Alvarez-Sabin J. Admission fibrinolytic profile is associated with symptomatic hemorrhagic transformation in stroke patients treated with tissue plasminogen activator. Stroke. 2004; 35:2123-2127. [PubMed: 15243150]

15. Selim M, Fink JN, Kumar S, Caplan LR, Horkan C, Chen Y, Linfante I, Schlaug G. Predictors of hemorrhagic transformation after intravenous recombinant tissue plasminogen activator: prognostic value of the initial apparent diffusion coefficient and diffusion-weighted lesion volume. Stroke. 2002; 33:2047-2052. [PubMed: 12154261]

16. Tong DC, Adami A, Moseley ME, Marks MP. Relationship between apparent diffusion coefficient and subsequent hemorrhagic transformation following acute ischemic stroke. Stroke. 2000; 31:2378-2384. [PubMed: 11022067]

17. Maier CM, Hsieh L, Crandall T, Narasimhan P, Chan PH. Evaluating therapeutic targets for reperfusion-related brain hemorrhage. Ann Neurol. 2006; 59:929-938. [PubMed: 16673393]

18. Trouillas P, von Kummer R. Classification and pathogenesis of cerebral hemorrhages after thrombolysis in ischemic stroke. Stroke. 2006; 37:556-561. [PubMed: 16397182]

19. Zaidat OO, Suarez JI, Santillan C, Sunshine JL, Tarr RW, Paras VH, Selman WR, Landis DM. Response to intra-arterial and combined intravenous and intra-arterial thrombolytic therapy in 
patients with distal internal carotid artery occlusion. Stroke. 2002; 33:1821-1827. [PubMed: 12105360]

20. Kidwell CS, Saver JL, Carneado J, Sayre J, Starkman S, Duckwiler G, Gobin YP, Jahan R, Vespa P, Villablanca JP, Liebeskind DS, Vinuela F. Predictors of hemorrhagic transformation in patients receiving intra-arterial thrombolysis. Stroke. 2002; 33:717-724. [PubMed: 11872894] 


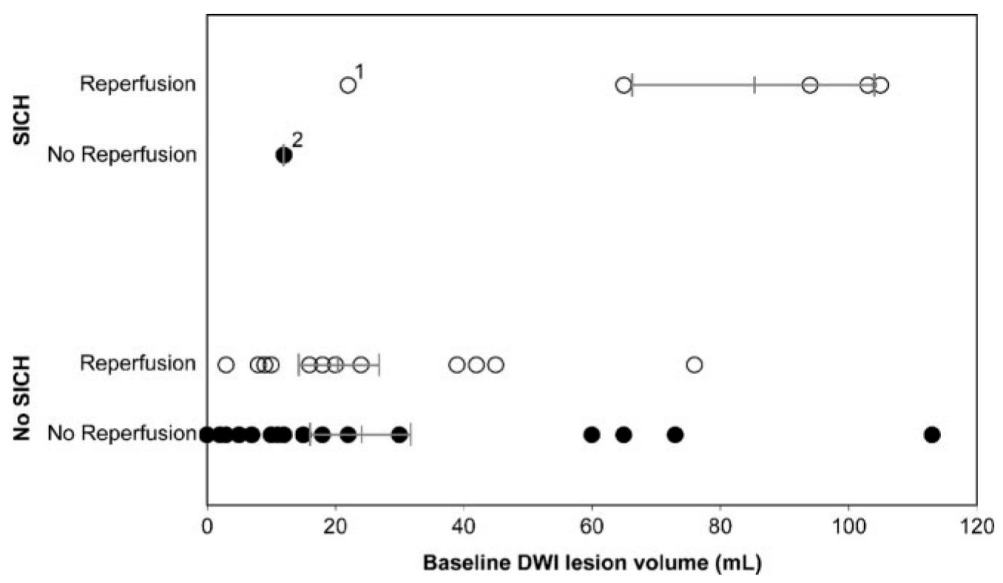

Figure.

This scatterplot displays the baseline DWI lesion volumes for 4 groups of patients: (1) patients with SICH and reperfusion (top row), (2) patients with SICH but without reperfusion (second row), (3) patients without SICH with reperfusion (third row), and (4) patients without SICH and without reperfusion (bottom row). Patients with reperfusion are marked with open circles $(\mathbf{)}$, and patients without reperfusion are marked with closed circles $(O)$. The bars indicate the mean \pm SEM for each group. Patients with SICH are characterized by reperfusion in the setting of large baseline DWI lesion volumes. The 2 patients with SICH who do not follow this pattern are numbered in the figure. Patient 1 had evidence of reperfusion on his follow-up scan without signs of hemorrhage. He developed an SICH during carotid endarterectomy performed the day after his stroke. Patient 2 had evidence of minor hemorrhagic transformation of his acute ischemic lesion on his follow-up MRI (4.5 hours after tPA therapy) associated with a 2 point increase in NIHSSS. 


\section{Table}

\section{Baseline Characteristics}

\begin{tabular}{lccc}
\hline Variable & $\begin{array}{c}\text { SICH } \\
(\mathbf{n}=7)\end{array}$ & $\begin{array}{c}\text { No SICH } \\
(\mathbf{n}=67)\end{array}$ & $\boldsymbol{P}$ \\
\hline Age, y & $78 \pm 12$ & $70 \pm 15$ & 0.21 \\
Male & $57 \%$ & $55 \%$ & 1.0 \\
History of cardiac disease & $57 \%$ & $40 \%$ & 0.44 \\
History of smoking & $29 \%$ & $43 \%$ & 0.69 \\
History of diabetes & $43 \%$ & $25 \%$ & 0.38 \\
History of stroke/TIA & $29 \%$ & $19 \%$ & 0.62 \\
History of hypertension & $89 \%$ & $58 \%$ & 0.24 \\
History of hyperlipidemia & $14 \%$ & $25 \%$ & 1.0 \\
Time to treatment, min & $307 \pm 27$ & $326 \pm 36$ & 0.20 \\
Serum glucose, mg/dL & $176 \pm 97$ & $132 \pm 50$ & 0.16 \\
Platelets, 1000/ $\mu \mathrm{L}$ & $235 \pm 76$ & $249 \pm 79$ & 0.66 \\
Systolic blood pressure pre-treatment, & $150 \pm 27$ & $150 \pm 20$ & 0.97 \\
mm Hg & & & \\
$\begin{array}{l}\text { Diastolic blood pressure pre-treatment, } \\
\text { mm Hg }\end{array}$ & $70 \pm 12$ & $76 \pm 16$ & 0.35 \\
NIHSSS at baseline & $17 \pm 6$ & $12 \pm 5$ & 0.02 \\
PWI lesion volume, mL & $130 \pm 76$ & $57 \pm 64$ & 0.01 \\
DWI lesion volume, mL & $62 \pm 40$ & $18 \pm 26$ & 0.03 \\
Early reperfusion ${ }^{*}$ & $83 \%$ & $35 \%$ & 0.04 \\
\hline
\end{tabular}

* Reperfusion status was assessed in 44 patients (6 patients with SICH and 38 patients without SICH). In 10 patients it could not be assessed because either the baseline or the follow-up PWI was technically inadequate, and in 20 patients it could not be assessed because the baseline PWI lesion volume was $<10 \mathrm{~mL}$ and therefore, by definition, reperfusion could not be assessed. 